\title{
A Retrospective Observational Analysis of Italian Reimbursed Healthcare for Patients on Hemodialysis and Treated for Chronic Kidney Disease-Associated Pruritus
}

\section{Silvia Calabria ( $\nabla$ calabria@fondazioneres.it )}

Fondazione ReS (Ricerca e Salute) - Research and Health Foundation

Giulia Ronconi

Fondazione ReS (Ricerca e Salute) - Research and Health Foundation

Carlo Piccinni

Fondazione ReS (Ricerca e Salute) - Research and Health Foundation

Letizia Dondi

Fondazione ReS (Ricerca e Salute) - Research and Health Foundation

\section{Enrico Cinconze}

Fondazione ReS (Ricerca e Salute) - Research and Health Foundation

\section{Antonella Pedrini}

Fondazione ReS (Ricerca e Salute) - Research and Health Foundation

Immacolata Esposito

Drugs and Health

\section{Alice Addesi}

Drugs and Health

\section{Lucio Manenti}

University Hospital of Parma

Filippo Aucella

Casa Sollievo della Sofferenza

Nello Martini

Fondazione ReS (Ricerca e Salute) - Research and Health Foundation

\section{Research Article}

Keywords: Chronic Kidney Diseases, Pruritus, Clinical Practice Pattern, Public Health Practice, Administrative Database

Posted Date: February 15th, 2022 
DOI: https://doi.org/10.21203/rs.3.rs-1196990/v1

License: (c) (1) This work is licensed under a Creative Commons Attribution 4.0 International License. Read Full License 


\section{Abstract}

Background. Chronic kidney disease-associated pruritus (CKD-aP) frequently affects patients on hemodialysis and still needs an effective treatment. This retrospective observational study characterized patients on hemodialysis potentially affected or not by CKD-aP and described the use of CKD-aP-related treatments and integrated healthcare costs, from the perspective of the Italian National Health Service (INHS).

Methods. Through the cross-linkage of the Italian administrative healthcare data collected in the database of Fondazione ReS (Ricerca e Salute), patients undergoing in-hospital/outpatient hemodialysis from $01 / 01 / 2015$ to $12 / 31 / 2017$ and for at least years were selected. Subjects with pruritus due to other causes (i.e. chronic liver disease/cirrhosis/systemic lupus erythematosus/arterial vasculitis/skinsubcutaneous tissue disease-inflammation) were excluded. CKD-aP/non-CKD-aP cohorts were created based on the presence/absence of CKD-aP-related treatment (based on common clinical practice and guidelines) supplies, characterized (demographics, comorbidities) and assessed in terms of CKD-aPrelated treatments reimbursed by the INHS and mean healthcare costs per capita paid by the INHS.

Results. Of 1,239 patients on hemodialysis for $\geq 2$ years ( $20.2 \%$ of all hemodialysis subjects), after having applied exclusion criteria, CKD-aP were 218 (17.6\%), non-CKD-aP were 1,021. Both cohorts were mostly males and elderly, but CKD-aP one was older and affected by more comorbidities. One-year before and after the index hemodialysis, of CKD-aP patients: CKD-aP-related treatments were supplied to $58.1 \%$ and $65.1 \%$, of which $>50 \%$ received chronic antihistamines (mostly cetirizine), $10 \%$ gabapentin and $1.4 \%$ received the ultraviolet light therapy. Calculated by mean, the annual cost for the healthcare reimbursed by the INHS per CKD-aP and non-CKD-aP patient was $€ 37,065$ and $€ 35,988$, respectively. Hemodialysis accounted for $>60 \%$ of hospitalization expenses and $>77 \%$ of outpatient care costs.

Conclusions. Due to the type of information collected in Italian administrative databases, the true prevalence of CKD-aP in the Italian hemodialysis population and the costs reimbursed by the INHS for the related healthcare resulted quite underestimated. Interestingly, high-efficiency dialytic therapies performed to CKD-aP patients seemed to largely weigh on the overall mean annual cost. These findings suggest that appropriate and effective treatments for this condition might offer cost offsets.

\section{Background}

Chronic kidney disease associated pruritus (CKD-aP) is a generalized persistent and refractory itching, frequently affecting patients on hemodialysis, also known as uremic pruritus, though its etiology has been found independent from uremia ${ }^{1,2}$. The most recent Dialysis Outcome and Practice Patterns Study (DOPPS) showed that patients in hemodialysis affected by at least moderate itchy skin ranged from $26 \%$ in Germany to 48\% in the United Kingdom in 2012-2015 analysis period and seemed not to increase over years ${ }^{2}$. Prevalence of CKD-aP seems to increase with the severity of the $C_{K D}{ }^{3}$ and with the time patients undergo hemodialysis ${ }^{4}$. CKD-aP occurs generally with dry skin and superimposable complications often 
tend to develop ${ }^{5}$. The negative symptom burden associated to CKD-aP is high and consists in discomfort, fatigue, poor sleep quality and depression ${ }^{3}$. Overall, the more severe the pruritus, the lower the mental and physical health status of patients affected by CKD-aP is ${ }^{3}$.

To date, the pathophysiology of CKD-aP is probably multifactorial but still unknown, therefore no causative or etiology-specific treatments have been established ${ }^{6,7}$. In case of CKD-aP, the European guidelines on chronic pruritus recommend the use of topical therapy (only capsaicin) and of systemic treatments ${ }^{7}$. These are antihistamines, both the first-generation (by modifying the dose accordingly in vulnerable populations) and the second-generation (although conventional doses in internal diseases have not proven effectiveness), corticosteroids (in selected cases and only for short-term use), the kopioid receptor agonist nalfurafine, gabapentin, pregabalin and, sporadically, thalidomide. Among the alternative non-pharmacological options, the ultraviolet (UV) phototherapy is well established for treating pruritus, especially the narrowband UVB ${ }^{7}$. Special populations (i.e. elderly, pregnancy and children) with $\mathrm{CKD}-\mathrm{aP}$ require particular attention and their treatment needs to be tailored case by case. Finally, according to the British Association of Dermatologists' guideline, which does not give specific recommendations, the only definitive treatment for CKD-aP is renal transplantation ${ }^{6}$. Besides the critical unmet need of effective and CKD-aP-specific therapies, unfortunately, CKD-aP is highly underreported by patients and overlooked by healthcare professionals ${ }^{1,2,8}$, mostly because of the lack of knowledge of its pathophysiology and the long-term outcomes ${ }^{9}$. Therefore the interest of health care policy makers and payers remains scarce and the production of evidence are not solicited, resulting in still many gaps in evidence, from epidemiological to cost analysis data.

This retrospective observational study of the Italian administrative healthcare data collected in the Fondazione Ricerca e Salute (ReS)'s database aimed to describe the population undergoing hemodialysis and potentially affected or not by CKD-aP, and to evaluate their healthcare resource consumption and the consequent economic burden, from the perspective of the Italian National Health Service (INHS).

\section{Methods}

\section{Data source}

This study originates from the cross linkage of the administrative healthcare databases owned by several Italian local and regional Health Authorities (HAs) and routinely collected in the ReS database under specific agreements. The record linkage is performed based on a unique anonymized identity number, according to the current European privacy legislation. These are the data HAs periodically convey to the Italian Ministry of Health for reimbursement purposes. The INHS is a universal coverage single-payer healthcare system, thus the healthcare data collected by local and regional databases could potentially represent the healthcare of all the beneficiaries of Italy. Although the ReS database cannot collect all the Italian administrative healthcare databases, it has been analyzed since 2018 for several observational studies on various clinical questions and fields and for institutional purposes ${ }^{10-12}$, since it has been 
demonstrated to be representative of the Italian population through a comparison of the age distributions provided by the Italian Institute of Statistics (ISTAT) ${ }^{13}$. The demographic database contains age, gender, local HA of residency and disease waiver claim for INHS patients' co-payment. The pharmaceutical database consists of all drugs reimbursed by the INHS and supplied from both local and hospital pharmacies. Active substances can be analyzed based on marketing code (AIC), Anatomical Therapeutic Chemical code (World Health Organization's ATC classification), dose, number of packages and dispensing date. The hospitalization database is analysable through in-hospital diagnoses and procedures accessible through the hospital discharge forms of both ordinary and daily hospitalizations (according to the current Italian version of the 9th version of the International Classification of Disease Clinical Modification - ICD-9-CM) ${ }^{14}$. The outpatient specialist care database is composed by examinations, diagnostics and invasive/non-invasive procedures and is analyzed based on the current national classification system. In addition, since Italian administrative healthcare databases were established for reimbursement purposes, all direct costs paid by the INHS related to the healthcare resource consumptions are included. The ReS database is physically placed into Cineca's supercomputers ${ }^{15}$. This collaboration guarantees compliance with international standard certifications of quality and safety of the data management. This is a retrospective observational study of Italian administrative healthcare data which have been analyzed in an aggregated form after their anonymization at the source, according to the specific agreements with the regional and local HAs, owners of the data, and to the European privacy laws. For these reasons and because of the institutional purposes of this study, neither informed consent nor ethical approval were applicable.

Finally, this study was not supported by experiments on humans and/or use of human tissue samples, therefore no relevant guidelines and regulations had to be followed for the correct performance of this study.

\section{Cohort selection}

Starting from the ReS database, the population with at least an in-hospital or outpatient hemodialysis procedure (ICD-9-CM code 39.95) during the 3-year accrual period (2015-2017) were identified. The beneficiaries captured by the ReS database corresponded to about $15 \%$ of the population resident in Italy according to the ISTAT's data ${ }^{13}$. The most recent date of hemodialysis procedure represented the index date. After having selected only patients with at least a 2-year ongoing hemodialysis treatment before the index date, those potentially affected by diseases that generally cause pruritus, such as chronic liver disease/cirrhosis, systemic lupus erythematosus, arterial vasculitis and disease/inflammatory conditions of the skin and subcutaneous tissue, from 2013 were excluded (for identification criteria, see

supplementary table 1). The final population was categorized according to the presence/absence of CKDaP-related treatments in 180 days before and/or after the index date: gabapentin, pregabalin, thalidomide and antihistamines and the UV phototherapy (for specific codes, see supplementary table 2). CKD-aPrelated treatments were selected according to common clinical practice and international guidelines' recommendations ${ }^{6,7}$. The time span of 180 days before and/or after the index date was chosen in order 
to be as accurate as possible in potentially associating CKD-aP and the specific treatments supplies. The two cohorts, are hereinafter called "CKD-aP" and "non-CKD-aP".

\section{Epidemiological and clinical characterization}

The cohorts were described in terms of age and gender at the index date. Moreover, in 2 years before the index date potential CKD-aP-related comorbidities (anemia, xerosis cutis, hyperparathyroidism, hypercalcemia, hyperuricemia and hyperphosphatemia) and other comorbidities of interest (hypothyroidism, diabetes mellitus, depression, arterial hypertension, viral hepatopathies and coronary artery disease - CAD) were assessed (for criteria, see supplementary table 3 ).

\section{$C K D-a P$ related treatments}

The CKD-aP related treatments reimbursed by the INHS were searched among pharmaceuticals (i.e. gabapentin, pregabalin, thalidomide and antihistamines), in hospital discharges and outpatient specialist care (i.e. UV therapy) databases. The therapeutic pattern was described in the CKD-aP cohort within 1 year before and after the index date, to be consistent with the other follow-up analyses. CKD-aP related treatments' consumption was analyzed in terms of DDD (defined daily dose - the assumed average maintenance daily dose of a drug used in its main indication), number of drug packages for drugs and UV phototherapy procedures, and portion of patients supplied with a CKD-aP related treatment at least once in 1 year before or after the index date.

\section{Healthcare integrated costs}

One-year follow-up healthcare costs were assessed for CKD-aP and non-CKD-aP patients. Moreover, in order to evaluate the average annual costs through an even more realistic perspective, each CKD-aP patient (case) was matched with a non-CKD-aP subject with the same demographic characteristics (age, gender, residency) (control), through a ratio 1:1. Only direct costs paid by the INHS and due to the healthcare resources' consumption, such as reimbursed pharmaceuticals, hospitalizations and outpatient specialist services are recorded in the Italian administrative databases. Specifically, the pharmaceutical costs were extrapolated by the gross expenditure for drugs supplied by local pharmacies, and by the real hospital price (inclusive of VAT) for drugs supplied by hospital pharmacies. The in-hospital expenditure was derived by the DRG (diagnosis related group) system tariffs. The DRG classification is used to estimate the hospitalization and the in-hospital stay costs per patient, for the INHS reimbursement. Each DRG code corresponds to all in-hospital cares (from the admission to the discharge) in their entirety and complexity, without distinguishing single performed services. Outpatient diagnostics and invasive/noninvasive procedures were assessed through the current national tariffs. Healthcare costs are shown as mean expenditure per capita for each database and for the overall integrated cost, and as percentage distributions. Pharmaceuticals expenses were split into CKD-aP-related treatments and "other drugs", which consisted in all drugs different from the CKD-aP-related ones. 
An individual matched pair case-control analysis was performed in order to assess the average annual cost by administrative healthcare database and overall, through an even more realistic perspective. Matched variables were gender, age and LHU of residency. Cases were people potentially affected by CKD-aP, while controls were those not affected by CKD-aP, according to the categorization by presence or absence of CKD-aP-related treatments.

Through administrative databases it is possible to analyse huge populations (millions of inhabitants), thus the identified target cohorts, although numerically small, can be considered large enough to assume that minimal differences are statistically significant for their conventional level $(p<0.05)$, even without any related convincing or plausible clinical meaning. For this reason, we have mostly avoided the use of detailed $p$ values and have described nominal differences.

All analyses were performed by means of Oracle SQL Developer Italian version 18.1.0.095, California, United States.

\section{Results}

\section{Epidemiological and clinical characterization}

From the ReS population in the three-year accrual period, 6,147 patients were treated at least once with hemodialysis, both in the in-hospital and outpatient settings (figure 1).

Of these, 1,589 subjects (25.9\%) were treated for at least 2 years. After the exclusion of patients potentially suffering from other diseases frequently causing pruritus, the final population was split into the CKD-aP cohort $(n=218)$ and the non-CKD-aP one $(n=1,021)$. Both cohorts were mostly males and elderly. Percentage distributions by age group showed prevalence increased with age, reaching a peak at 70-79 years (table 1). CKD-aP patients resulted slightly older than non-CKD-aP ones.

The analysis of comorbidities potentially related to CKD-aP showed that hyperphosphatemia, hyperparathyroidism, anaemia and hyperuricemia affected both cohorts in the same descending order of frequency (table 1). CKD-aP patients were slightly more affected by all of them, while hyperuricemia resulted more frequent in non-CKD-aP cohort. Among the other comorbidities of interest, arterial hypertension, diabetes mellitus, hypothyroidism, depression, CAD and viral hepatopathies were in the same descending order of frequency in both cohorts (table 1).

Table 1 - Baseline characteristics of patients with/without chronic kidney disease-associated pruritus 


\begin{tabular}{|c|c|c|}
\hline & $\begin{array}{l}\text { CKD-aP patients } \\
(n=218)\end{array}$ & Non-CKD-aP patients $(n=1,021)$ \\
\hline Males (n; \%) & $121 ; 55.5$ & $583 ; 57.1$ \\
\hline Median age (Q1; Q3) & $71(61 ; 80)$ & $68(53 ; 79)$ \\
\hline Mean age ( \pm standard deviation) & $69( \pm 13)$ & $66( \pm 15)$ \\
\hline \multicolumn{3}{|l|}{ Distribution by age group (\%) } \\
\hline$<18$ & 0.0 & 0.2 \\
\hline $18-29$ & 0.9 & 0.8 \\
\hline $30-39$ & 1.4 & 3.8 \\
\hline $40-49$ & 4.6 & 11.6 \\
\hline $50-59$ & 13.8 & 15.3 \\
\hline $60-69$ & 27.1 & 23.0 \\
\hline 70-79 & 28.0 & 25.8 \\
\hline$\geq 80$ & 24.3 & 19.6 \\
\hline Total & 100.0 & 100.0 \\
\hline \multicolumn{3}{|c|}{ Potential CKD-aP-related comorbidities (n; \%) } \\
\hline Hyperphosphatemia & $150 ; 68.8$ & $623 ; 61.0$ \\
\hline Hyperparathyroidism & $102 ; 46.8$ & $437 ; 42.8$ \\
\hline Anemia & $81 ; 37.2$ & $364 ; 35.7$ \\
\hline Hyperuricemia & $36 ; 16.5$ & $178 ; 17.4$ \\
\hline Hypercalcemia & - & - \\
\hline Xerosis cutis & - & - \\
\hline \multicolumn{3}{|l|}{ Other comorbidities of interest $(\mathrm{n} ; \%)$} \\
\hline Arterial hypertension & $162 ; 74.3$ & $732 ; 71.7$ \\
\hline Diabetes mellitus & $57 ; 26.1$ & $195 ; 19.1$ \\
\hline Hypothyroidism & $41 ; 18.8$ & $138 ; 13.5$ \\
\hline Depression & $31 ; 14.2$ & $85 ; 8.3$ \\
\hline Coronary artery disease & $21 ; 9.6$ & $86 ; 8.4$ \\
\hline Viral hepatopathies & $2 ; 0.9$ & $5 ; 0.5$ \\
\hline
\end{tabular}


CKD-aP: chronic kidney disease-associated pruritus

\section{CKD-aP related treatments}

Table 2 shows that $58.3 \%$ and $65.1 \%$ of CKD-aP patients received at least one CKD-aP related drug during one year before or after the index date, respectively. In both observation periods, about $10 \%$ of the cohort was treated with gabapentin and around $50 \%$ was supplied with antihistamines (cetirizine was the most supplied: $24.3 \%$ ). The mean consumption of antihistamines (4.6 packs per patient) was in line with an annual chronic use. For all CKD-aP related drugs, the annual mean consumption in terms of number of packages and DDD during one year before and after the index date were very similar. The $1.4 \%$ of CKD-aP patients were treated with UV phototherapy at least once over one year before or after the index date. On average, the UV phototherapy was performed 2 times in the previous year and 4 times in the subsequent one to each patient.

Table 2 - Prescribing patterns of chronic kidney disease-associated pruritus related treatments one year before/after the index date.

\begin{tabular}{|c|c|c|c|c|c|c|}
\hline \multirow{3}{*}{$\begin{array}{l}\text { CKD-aP related } \\
\text { treatments }\end{array}$} & \multicolumn{3}{|l|}{ - 365 days } & \multicolumn{3}{|c|}{ + 365 days } \\
\hline & $\begin{array}{l}\text { Patients; \% } \\
\text { on CKD-aP } \\
\text { cohort ( } n= \\
218)\end{array}$ & $\begin{array}{l}\text { Mean } n^{\circ} \text { of } \\
\text { packages } \\
\text { per patient } \\
\text { treated }\end{array}$ & $\begin{array}{l}\text { Mean } \\
\text { DDD per } \\
\text { patient } \\
\text { treated }\end{array}$ & $\begin{array}{l}\text { Patients; } \\
\% \text { on } \\
\text { CKD-aP } \\
\text { cohort }\end{array}$ & $\begin{array}{l}\text { Mean } n^{\circ} \text { of } \\
\text { packages } \\
\text { per patient } \\
\text { treated }\end{array}$ & $\begin{array}{l}\text { Mean } \\
\text { DDD per } \\
\text { patient } \\
\text { treated }\end{array}$ \\
\hline & & & & \multicolumn{3}{|l|}{$(n=218)$} \\
\hline Gabapentin & $23 ; 10.6$ & 4.7 & 21.0 & $20 ; 9.2$ & 5.4 & 25.5 \\
\hline Pregabalin & 0 & - & - & 0 & - & - \\
\hline Thalidomide & 0 & - & - & 0 & - & - \\
\hline Antihistamines & $108 ; 49.5$ & 4.6 & 93.6 & $122 ; 56.0$ & 4.2 & 88.2 \\
\hline $\begin{array}{l}\text { Ultraviolet light } \\
\text { therapy }\end{array}$ & $3 ; 1.4$ & 2.0 & NA & $3 ; 1.4$ & 4.0 & NA \\
\hline $\begin{array}{l}\text { At least one } \\
\text { CKD-aP related } \\
\text { treatment }\end{array}$ & \multicolumn{3}{|l|}{$127 ; 58.3$} & \multicolumn{3}{|l|}{$142 ; 65.1$} \\
\hline
\end{tabular}

CKD-aP: chronic kidney disease-associated pruritus; DDD: defined daily dose; NA: not available

\section{Healthcare integrated costs}

The cost analysis (table 3 ) estimated that, on average, during the follow-up year, the INHS paid $€ 37,065$ for a CKD-aP patient, $€ 31,286$ for a control and $€ 35,988$ for a non-CKD-aP subject. In all cohorts, the mean costs associated to each administrative healthcare database showed similar percentage distribution on the overall expenditure: about the $80 \%$ was due to outpatient specialist services, about 
$10 \%$ to pharmaceuticals and to hospitalizations. Concomitant drugs determined the most of the expenses for pharmaceuticals. Hemodialysis accounted for around $80 \%$ of the outpatient care cost and for more than $60 \%$ of the hospitalization expenditure.

Table 3 - Healthcare integrated costs per patient with/without chronic kidney disease-associated pruritus, within one year of follow-up 


\begin{tabular}{|c|c|c|c|}
\hline \multirow{3}{*}{$\begin{array}{l}\text { Administrative } \\
\text { healthcare database } \\
\text { Specific cost item }\end{array}$} & $\begin{array}{l}\text { CKD-aP patients } \\
(n=218) \text { - cases }\end{array}$ & $\begin{array}{l}\text { Non-CKD-aP patients } \\
(n=218)-\text { controls }\end{array}$ & $\begin{array}{l}\text { Non-CKD-aP patients } \\
(n=1,021)\end{array}$ \\
\hline & $\begin{array}{l}\text { Mean cost }(€) \text { per } \\
\text { capita }\end{array}$ & $\begin{array}{l}\text { Mean cost }(€) \text { per } \\
\text { capita }\end{array}$ & $\begin{array}{l}\text { Mean cost }(€) \text { per } \\
\text { capita }\end{array}$ \\
\hline & $\begin{array}{l}\text { (\% on overall/ } \\
\text { administrative } \\
\text { database } \\
\text { expenditure) }\end{array}$ & $\begin{array}{l}\text { (\% on overall/ } \\
\text { administrative } \\
\text { database } \\
\text { expenditure) }\end{array}$ & $\begin{array}{l}\text { (\% on overall/ } \\
\text { administrative } \\
\text { database } \\
\text { expenditure) }\end{array}$ \\
\hline Pharmaceuticals & 3667 (9.9) & $2721(8.7)$ & $3430(9.5)$ \\
\hline CKD-aP-related drugs & $20(0.5)$ & $1(0.0)$ & $<1(0.0)$ \\
\hline Other drugs & 3647 (99.5) & 2720 (99.9) & $3430(100.0)$ \\
\hline Hospitalizations & 3590 (9.7) & $3129(10.0)$ & $3640(10.1)$ \\
\hline Hemodialysis & $2238(62.3)$ & $1980(63.3)$ & 2413 (66.3) \\
\hline $\begin{array}{l}\text { Outpatient specialist } \\
\text { services }\end{array}$ & $29809(80.4)$ & $25437(81.3)$ & $28917(80.4)$ \\
\hline Ultraviolet light therapy & $<1(0.0)$ & $<1(0.0)$ & $0(0.0)$ \\
\hline Hemodialysis & $23239(77.9)$ & 19926 (78.3) & $22697(78.4)$ \\
\hline $\begin{array}{l}\text { Bicarbonate } \\
\text { hemodialysis with } \\
\text { biocompatible } \\
\text { membrane }\end{array}$ & $13763(46.2)$ & $12187(47.9)$ & $13565(46.9)$ \\
\hline Other hemodiafiltration & $5465(18.3)$ & $4890(19.2)$ & $5296(18.3)$ \\
\hline Hemodiafiltration & $1680(5.6)$ & $1,364(5.4)$ & $2083(7.2)$ \\
\hline $\begin{array}{l}\text { Hemodiafiltration, } \\
\text { limited assistance }\end{array}$ & $1286(5.6)$ & 745 (2.9) & $619(2.1)$ \\
\hline $\begin{array}{l}\text { Acetate and bicarbonate } \\
\text { hemodialysis }\end{array}$ & $783(2.6)$ & $548(2.1)$ & $775(2.7)$ \\
\hline Hemofiltration & $91(0.3)$ & $1(0.0)$ & $126(0.4)$ \\
\hline $\begin{array}{l}\text { Hemodialysis - } \\
\text { hemofiltration }\end{array}$ & $89(0.3)$ & $88(0.3)$ & $160(0.5)$ \\
\hline $\begin{array}{l}\text { Acetate and bicarbonate } \\
\text { hemodialysis, limited } \\
\text { assistance }\end{array}$ & $81(0.3)$ & $104(0.4)$ & $72(0.2)$ \\
\hline $\begin{array}{l}\text { Home acetate and } \\
\text { bicarbonate } \\
\text { hemodialysis }\end{array}$ & $0(0.0)$ & $0 ; 0.0$ & $<1(0.0)$ \\
\hline Total cost & 37065 (100.0) & $31286 ; 100.0$ & 35988 (100.0) \\
\hline
\end{tabular}


CKD-aP: chronic kidney disease-associated pruritus

\section{Discussion}

In this observational study, $17.6 \%$ of patients on hemodialysis therapy in Italy from 2015 to 2017 were potentially affected by CKD-aP. This prevalence is hard to compare with literature, which originates from qualitative studies using different ways of measuring CKD-aP and produced inconsistent data 2,16 . Nevertheless, the DOPPS phase 5 (2012-2015) ${ }^{2}$ found from 5 to $20 \%$ of hemodialysis patients at least moderately bothered by pruritus. Facing the absence of a specific healthcare service identifying the CKDaP and of clinical information (e.g. coming from dialysis registries, general practitioner's database, laboratory values), we identified patients by means of the reimbursed supply of gabapentin, pregabalin, thalidomide, antihistamines, recommended by the current guidelines ${ }^{6,7}$, and performance of the UV phototherapy. The administrative healthcare databases record only the healthcare reimbursed by the INHS. Nevertheless, since none of them is specific for CKD-aP, we excluded some concomitant conditions (see methods) that frequently cause pruritus and can be treated with the aforementioned therapies 6,7 . Particularly, antihistamines corresponded to the most common therapeutic strategy for CKD-aP in the Italian clinical practice ${ }^{2}$. Their chronic supply is reimbursed by the INHS in presence of a chronic severe condition (e.g. the excluded diseases), but also in case of seasonal allergic rhino-conjunctivitis that needs long-term treatment with antihistamines ${ }^{17}$, which is, unfortunately, hard to identify within the administrative databases. Therefore, inevitably the few patients affected by this particular condition and coincidently by CKD felt into our CKD-aP cohort. Actually, we selected the CKD-aP patients within the limits of Italian administrative healthcare databases. CKD-aP subjects identified by this study were

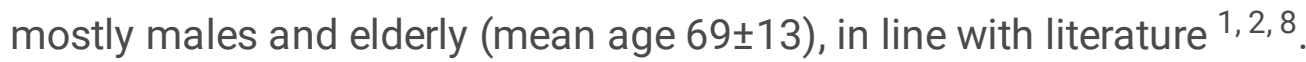

To date, very few researches have examined the prevalence, characteristics and outcomes of CKD-aP ${ }^{16}$. Some of them have raised the critical frequent tendency of underreporting pruritus by patients and overlooking it by general practitioners, nephrologists and other healthcare professionals ${ }^{1,2,8}$. The lack of renal functioning information and of possible previous lines of treatment prevents us from defining the severity of CKD. However, based on a study evaluating the reasons for underreporting pruritus in CKD patients ${ }^{8}$, we can suppose that most of them received healthcare by the INHS in a very discomfort condition, probably affected by at least a moderate form of CKD-aP.

The etiopathogenesis of CKD-aP is still unknown and probably multifactorial, but some concomitant metabolic disorders (e.g. related to serum calcium, phosphorus and ferritin, parathormone, hemoglobin and albumin) have been frequently highlighted, though causality still has not been confirmed ${ }^{1-4}$. In this study, the prevalence of hyperphosphatemia, hyperparathyroidism and anemia resulted higher in the CKDaP cohort than in the non-CKD-aP one. Higher percentages of patients affected by the other comorbidities of interest were also found in the CKD-aP cohort. It is acknowledged that subjects suffering from CKD-aP are characterized by low physical and mental status, but it is still unclear among the concomitant 
diseases which ones are involved in the etiopathogenesis, or carried by patients from the pre-dialysis period or only consequences, since no supporting evidence still have been provided.

The CKD-aP symptom burden is high and it is very important to readily reduce it. Since an established and effective cure does not yet exist, clinicians' attempt is based on the available evidence, even if not proven. The most updated guidelines ${ }^{6,7}$ do not recommend a clear management, but recent studies and reviews $2,16,18,19$ showed a tendency to follow a stepwise approach. It consists ideally in first trying to reach dialysis clearance target or mineral and bone ones; then to treat skin xerosis, if present, with emollient creams; if pruritus persists, to prescribe gabapentin or pregabalin. The dialytic approach will be discussed in the cost analysis section. Therapies for mineral and bone disorders are not evaluable through administrative databases, as well as the topical treatments ${ }^{7}$.

In real life, nephrologists actually prescribe first and second generation antihistamines to treat CKD-aP, without further intervention. More than half of the medical directors interviewed by Rayner and colleagues prescribed oral and topical antihistamines as first choice for pruritus ${ }^{2}$. In our study, antihistamines were dispensed to about $50 \%$ of CKD-aP patients, both one year before and after the index date. Since diagnoses of dermatitis or autoimmune pathologies possibly related to the prescription of antihistamines were excluded, we can state that the antihistamines supply were the greater marker of Italian CKD-aP patients in the ReS database. Moreover, in the same study ${ }^{2}$, less than $10 \%$ of the interviewed patients affected by pruritus were treated with gabapentin or pregabalin. Particularly, even if gabapentin is, by now, the only drug worldwide marketed with the highest evidence against CKD-aP ${ }^{20}$, in Italy it is not used as first choice ${ }^{2}$. We found that gabapentin was supplied to $10 \%$ of CKD-aP patients. It is worth mentioning that its reimbursement is limited to specific cases of neuropathy ${ }^{21}$, without ever mentioning the CKD-aP, and to epilepsy out of this recommendation. Therefore, the portion of subjects treated with gabapentin in this study resulted underestimated. This analysis also assessed the use of pregabalin, but no hemodialysis patient received it charged to the INHS. Thalidomide is also recommended by current guidelines ${ }^{7}$, with a number of proposed mechanisms of action against pruritus, but it has not been reimbursed to any of our hemodialysis patients. UV phototherapy has shown dramatic improvement in pruritus ${ }^{16}$ and in this study it was performed, charged to the INHS, to $1.4 \%$ of CKD-aP patients. The doubling of frequency from 2 to 4 UV therapies after the index date could suggest that it was successful. Based on the most updated literature, 4 theories on the pathophysiologic mechanisms behind CKD-aP exist ${ }^{16}$ : peripheral neuropathy, immune system dysregulation, opioid imbalance, and toxin deposition. The identification of comorbidities is essential to identify them and establish a therapeutic strategy to reduce or eliminate these disorders. Promising novel therapies based on the opioid imbalance (difelikefalin, а к-opioid receptor antagonist) and the immune dysregulation theories are under evaluation ${ }^{19}$. Even if this analysis could evaluate only the recommended pharmacotherapies reimbursed by the INHS and evaluable through administrative databases, the amount of patients with CKD-aP treated with at least a therapy recommended for CKD-aP ( $58.1 \%$ before and $65.1 \%$ after index date) was close to what found by Rayner and colleagues (DOPPS phase 5) in $2017^{2}$ in hemodialysis patients with pruritus. 
The DOPPS phase 5 found that $68 \%$ of patients with moderate to extreme pruritus used topical treatments, $28 \%$ oral ones and the UV phototherapy was rarely prescribed. Overall, the most prescribed were antihistamines, gabapentin, sedatives and corticosteroids. Furthermore, the fact that the amount of patients treated at least once in the observational periods was not the $100 \%$ of the selected cohort deserves an explanation. Indeed, the selection was based on the presence of a CKD-aP treatment 180 days before and/or after the index date, while the free filled drug prescriptions were evaluated 365 days before or after the index date. Thus, both the algorithm and the time lapses justified the smaller amount in the observational periods.

On average, the annual direct economic impact on the INHS due to the healthcare resource consumption (i.e. reimbursed pharmaceuticals, hospitalizations and outpatient specialist services) was higher for a CKD-aP patient than for a non-CKD-aP one. These costs are quietly underestimated, mostly because administrative databases do not record the private purchase of healthcare services (for instance, selfmedication through over the counter drugs for CKD-aP seems very frequent ${ }^{3}$ ) and all indirect costs (e.g. those due to the loss of productivity or the caregiver support). Nevertheless, the cost analysis is crucial for understanding the possible pathway of CKD-aP patients and for comparing it with that of subjects undergoing hemodialysis but not experiencing pruritus. CKD-aP and non-CKD-aP cohorts similarly weighed to the INHS in terms of percentage distribution of the cost related to each database on the overall expenditure. Particularly, concomitant drugs corresponded with the entire pharmaceutical expenditure for the non-CKD-aP cohorts, while accounted for the $99.5 \%$ of the CKD-aP one. At the same time, the higher cost for other drugs in the CKD-aP cohort probably reflected a more unbalanced condition, which is not however possible to directly correlate with the CKD-aP itself only by means of the administrative healthcare data. Hemodialysis was predominately performed in the outpatient setting, as expected with respect to the Italian operating custom. Hemodiafiltration provided the highest cost. The performance of hemodialysis is considered critical for the successful limitation of pruritus after the therapy. This is demonstrated by the higher cost of the wide and heterogeneous hemodialysis approaches compared to that generated by the CKD-aP related treatments. The very low use of CKD-aP related therapies is, in turn, in line with the healthcare professionals' common stepwise management assessed by Rayner and colleagues ${ }^{2}$, that, in case of serious pruritus, tended to increase the dialysis dose before prescribing medications. Changing the hemodialytic method has been suggested as an approach to treat CKD-aP based on the toxin deposition theory, without however showing any real improvement ${ }^{16}$. Whereas, high-flux hemodialysis, hemodiafiltration with hemoperfusion and highpermeability hemodialysis have shown significant relief of uremic pruritus compared to the conventional hemodialysis ${ }^{20}$. Nevertheless, beyond the unclear effectiveness of the dialysis time increase ${ }^{16}$, Sukul and colleagues ${ }^{1}$ showed that the post dialysis recovery time from pruritus increases with the severity of the pruritus itself, contributing to add to the already high physical and psychological symptom burden and to missed treatments and withdrawal from dialysis, which was found significant among hemodialysis patients. Moreover, the potential loss of autonomy to reach the dialysis center, that inevitably causes the need for transport and increases the indirect costs related to dialysis, has been previously demonstrated in Italy ${ }^{22}$. Our findings about costs showed plausible heterogeneous 
approaches to CKD-aP patients, mainly characterized by a continuous research of an effective dialysis treatment. Particularly, the not negligible use of hemodiafiltration suggested that clinicians tried an upgrade from hemodialysis to hemodiafiltration, in hopes of controlling pruritus through a better purification, probably not always successfully. Thus, the discovery of an effective treatment would lead to recommend this shift as a following step and to savings on high efficiency dialytic therapies, which are the most contributing to the average total cost of a CKD-aP patient. Although only descriptive, this cost analysis integrated the very few evidence in the CKD-aP panorama.

\section{Strengths and limitations}

Limitations of the exclusive use of this type of data are several, other than the difficulty in identifying pruritus reliably associated to CKD, previously deepened. First of all, the absence of clinical data (e.g. dialysis vintage, dialysis dose), information on renal function and other relevant patient's characteristics could have contributed to quietly underestimate the cohort selection. The ReS database still does not link to other databases, such as dialysis registries, general practitioner's operating system, or those collecting laboratory outcomes or the private purchase (i.e. drugs or healthcare services totally in the charge of patients). Overall, some CKD-aP patients assisted by the general medicine, together with those who do not receive healthcare by the INHS, remain undetected by this study. Moreover, the CKD identification is possible only through diagnosis and procedure codes, particularly the dialysis therapy in Italy is detectable only by means of in-hospital or outpatient procedure codes, whose precision depends on healthcare professionals. By the way, administrative data can analyse a large and unselected sample size which reliably reflects the real population. Moreover, the accuracy of administrative healthcare databases in researching CKD patients has been demonstrated high ${ }^{23}$.

\section{Conclusions}

This analysis of the ReS database provided a picture of CKD-aP in hemodialysis patients variously distributed in Italy from 2015 to 2017. Even if the cohort potentially affected by CKD-aP resulted underestimated, because of the limitations described, this study has highlighted some current critical therapeutic strategies in the Italian nephrology settings. It was confirmed the already observed widespread use of antihistamines in Italy, which are recommended by guidelines for CKD-aP, but still lack of enough evidence to support such a common use. Moreover, very few CKD-aP patients have been treated with gabapentin that, in contrast, has to date the strongest evidence for the CKD-aP treatment, but cannot be reimbursed by the INHS. Even more important, an extensive use of high-efficiency haemodialytic therapies, inferred by the very high impact on the hemodialysis costs, suggested that, probably, hemodialysis was chosen as first step in the management of CKD-aP. Our findings proved this wide use was too expensive and should be delayed in favour of strategies with strongest evidence. CKDaP patients still has significant unmet needs. Healthcare policy makers and payers must favour researches to improve treatments, but this effort accomplishes above all through the awareness of the healthcare professionals and patients themselves. Indeed, facing the poor understanding of the pathophysiology of CKD-aP and lack of knowledge of its long-term outcomes, clinicians still tend to 
consider the pruritus a secondary aspect of dialysis subjects, while patients still ignore the potential correlation of this symptom with their condition ${ }^{8}$. Raising the awareness and sharing information through updated guidelines and education initiatives for clinicians and patients are deserved. Larger and higher-quality trials are needed ${ }^{20}$ in order to bridge the gap between knowledge and patients' needs and priorities and to find an effective treatment as soon as possible. Meanwhile, clinicians are encouraged taking a detailed history of hemodialysis patients with chronic pruritus ${ }^{7,18}$, following the current evidence on the step approach and reducing the widespread use of expensive dialytic therapies.

\section{List Of Abbreviations}

CKD-aP: chronic kidney disease - associated pruritus

DOPPS: Dialysis Outcome and Practice Patterns Study

UV: ultraviolet

ReS: Ricerca e Salute

INHS: Italian National Health Service

LHU: Local Health Unit

ISTAT: Italian Institute of Statistics

AIC: Italian marketing code for drugs

ATC: anatomical chemical therapeutic classification

ICD-9-CM: International Classification of Disease $-9^{\text {th }}$ version - Clinical Modification

CAD: coronary artery disease

DDD: defined daily dose

VAT: value added tax

DRG: diagnosis related group

\section{Declarations}

Acknowledgements: not applicable

Ethics approval: This was a retrospective observational study of Italian administrative data which have been analyzed in an aggregated form after their anonymization at the source, according to the specific agreements with the Regional/Local Health Authorities, owners of the data, and to the European privacy 
laws. For these reasons and because of the institutional purposes of this study, ethical approval was not applicable.

Informed consent: This is a retrospective observational study of Italian administrative data which have been analyzed in an aggregated form after their anonymization at the source, according to the specific agreements with the Regional/Local Health Authorities, owners of the data, and to the European privacy laws. For these reasons and because of the institutional purposes of this study, informed consent was not applicable.

Consent for publication: not applicable

Availability of data and material: The datasets analysed during the current study are not publicly available and are not available from the corresponding author on reasonable request, because they are owned by the Italian Regional/Local Health Authorities who have not authorized Fondazione ReS to make them available.

Competing interests: LM declares its participation in the advisory boards of Vifor Pharma, GlaxoSmithKline and Galderma. FA declares its participation in the advisory board of Vifor Pharma. The other authors declare that they have no competing interests.

Funding: Financial support for this study was provided by an unconditional grant from Vifor Pharma, Glattbrugg, Switzerland. The funding agreement ensured the authors' independence in designing the study, interpreting the data, writing, and publishing the report.

Author Contributions: Conceptualization, S.C., G.R., L.D., C.P., A.P., L.M. and F.A.; data curation, L.D. and E.C.; formal analysis, G.R. and L.D.; funding acquisition, A.P., I.E., A.A. and N.M.; investigation, G.R., S.C., L.D and E.C..; methodology, S.C., G.R., L.D., L.M. and F.A.; project administration, I.E. and A.A.; software, L.D.; supervision, N.M.; validation, S.C., G.R., L.D., C.P., E.C., A.P., L.M. and F.A.; writing-original draft, S.C., L.M. and F. A.; writing-review and editing, S.C., L.M. and F.A.. All authors have read and agreed to the published version of the manuscript.

Code availability: Oracle SQL Developer version 18.1.0.095 (California, United States) was the software used in order to conduct all the analyses.

\section{References}

1. Sukul N, Karaboyas A, Csomor PA, et al. Self-reported Pruritus and Clinical, Dialysis-Related, and Patient-Reported Outcomes in Hemodialysis Patients. Kidney medicine 2021; 3: 42-53.e41. 2021/02/20. DOI: 10.1016/j.xkme.2020.08.011.

2. Rayner HC, Larkina $M$, Wang $M$, et al. International Comparisons of Prevalence, Awareness, and Treatment of Pruritus in People on Hemodialysis. Clin J Am Soc Nephrol 2017; 12: 2000-2007. DOI: $10.2215 /$ cjn.03280317. 
3. Ramakrishnan K, Bond TC, Claxton A, et al. Clinical characteristics and outcomes of end-stage renal disease patients with self-reported pruritus symptoms. Int J Nephrol Renovasc Dis 2013; 7: 1-12. 2014/01/01. DOI: 10.2147/ijnrd.s52985.

4. Kimata N, Fuller DS, Saito A, et al. Pruritus in hemodialysis patients: Results from the Japanese Dialysis Outcomes and Practice Patterns Study (JDOPPS). Hemodialysis international International Symposium on Home Hemodialysis 2014; 18: 657-667. 2014/04/29. DOI: 10.1111/hdi.12158.

5. Mettang T and Kremer AE. Uremic pruritus. Kidney Int 2015; 87: 685-691. 2014/01/10. DOI: 10.1038/ki.2013.454.

6. Millington GWM, Collins A, Lovell CR, et al. British Association of Dermatologists' guidelines for the investigation and management of generalized pruritus in adults without an underlying dermatosis, 2018. Br J Dermato/2018; 178: 34-60. 2018/01/24. DOI: 10.1111/bjd.16117.

7. Weisshaar E, Szepietowski JC, Dalgard FJ, et al. European S2k Guideline on Chronic Pruritus. Acta Derm Venereo/2019; 99: 469-506. 2019/04/02. DOI: 10.2340/00015555-3164.

8. Aresi G, Rayner HC, Hassan L, et al. Reasons for Underreporting of Uremic Pruritus in People With Chronic Kidney Disease: A Qualitative Study. J Pain Symptom Manage 2019; 58: 578-586.e572. 2019/06/23. DOI: 10.1016/j.jpainsymman.2019.06.010.

9. Ting SW, Fan PC, Lin YS, et al. Association between uremic pruritus and long-term outcomes in patients undergoing dialysis. J Am Acad Dermatol 2020; 83: 924-925. 2020/01/16. DOI: 10.1016/j.jaad.2020.01.011.

10. Piccinni C, Cevoli S, Ronconi G, et al. Insights into real-world treatment of cluster headache through a large Italian database: prevalence, prescription patterns, and costs. Expert Rev Clin Pharmacol 2021: 1-7. DOI: 10.1080/17512433.2021.1934448.

11. Maggioni AP, Dondi L, Andreotti F, et al. Prevalence, clinical impact and costs of hyperkalaemia: Special focus on heart failure. Eur J Clin Invest 2021: e13551. 2021/04/01. DOI: 10.1111/eci.13551.

12. Ronconi G, Dondi L, Calabria S, et al. Real-world Prescription Pattern, Discontinuation and Costs of Ibrutinib-Naïve Patients with Chronic Lymphocytic Leukemia: An Italian Healthcare Administrative Database Analysis. Clin Drug Investig 2021 2021/05/26. DOI: 10.1007/s40261-021-01044-3.

13. Istituto Nazionale di Statistica and ISTAT. Resident population by age, sex and marital status http://demo.istat.it/index_e.html (2015, accessed 05 June 2020).

14. Ministero del Lavoro and della Salute e delle Politiche Sociali. Classificazione delle malattie, dei traumatismi, degli interventi chirurgici e delle procedure dagnostiche e terapeutiche. Versione italiana della ICD9-CM. 2007.

15. CINECA - Interuniversity Consortium, https://www.cineca.it/ (accessed 12/22/2021).

16. Verduzco HA and Shirazian S. CKD-Associated Pruritus: New Insights Into Diagnosis, Pathogenesis, and Management. Kidney international reports 2020; 5: 1387-1402. 2020/09/22. DOI: 10.1016/j.ekir.2020.04.027.

17. Agenzia Italiana del Farmaco. Nota 89. GU Serie Generale n.259 del 04-11-2004 2004. 
18. Arzhan S, Roumelioti ME and Unruh ML. Itch and Ache on Dialysis: New Approaches to Manage Uremic Pruritus and Restless Legs. Blood Purif 2020; 49: 222-227. DOI: 10.1159/000504081.

19. Trachtenberg AJ, Collister $D$ and Rigatto $C$. Recent advances in the treatment of uremic pruritus. Curr Opin Nephrol Hypertens 2020; 29: 465-470. 2020/08/03. DOI: 10.1097/mnh.0000000000000625.

20. Simonsen E, Komenda P, Lerner B, et al. Treatment of Uremic Pruritus: A Systematic Review. Am J Kidney Dis 2017; 70: 638-655. 2017/07/20. DOI: 10.1053/j.ajkd.2017.05.018.

21. Agenzia Italiana del Farmaco. Nota 4, https://www.aifa.gov.it/nota-04 (2017, accessed 20 July 2021).

22. Gillespie F, Amicosante AMV, Lo Scalzo A, et al. Agenas - HTA Report Adaptation - "Valutazione HTA delle Modalità di dialisi in Italia". 2015.

23. van Oosten MJM, Logtenberg SJJ, Edens MA, et al. Health claims databases used for kidney research around the world. Clinical Kidney Journal 2020; 14: 84-97. DOI: 10.1093/ckj/sfaa076.

\section{Figures}


Patients undergoing at least an in-hospital/outpatient hemodialysis procedure from 2015 to $2017: 6147$

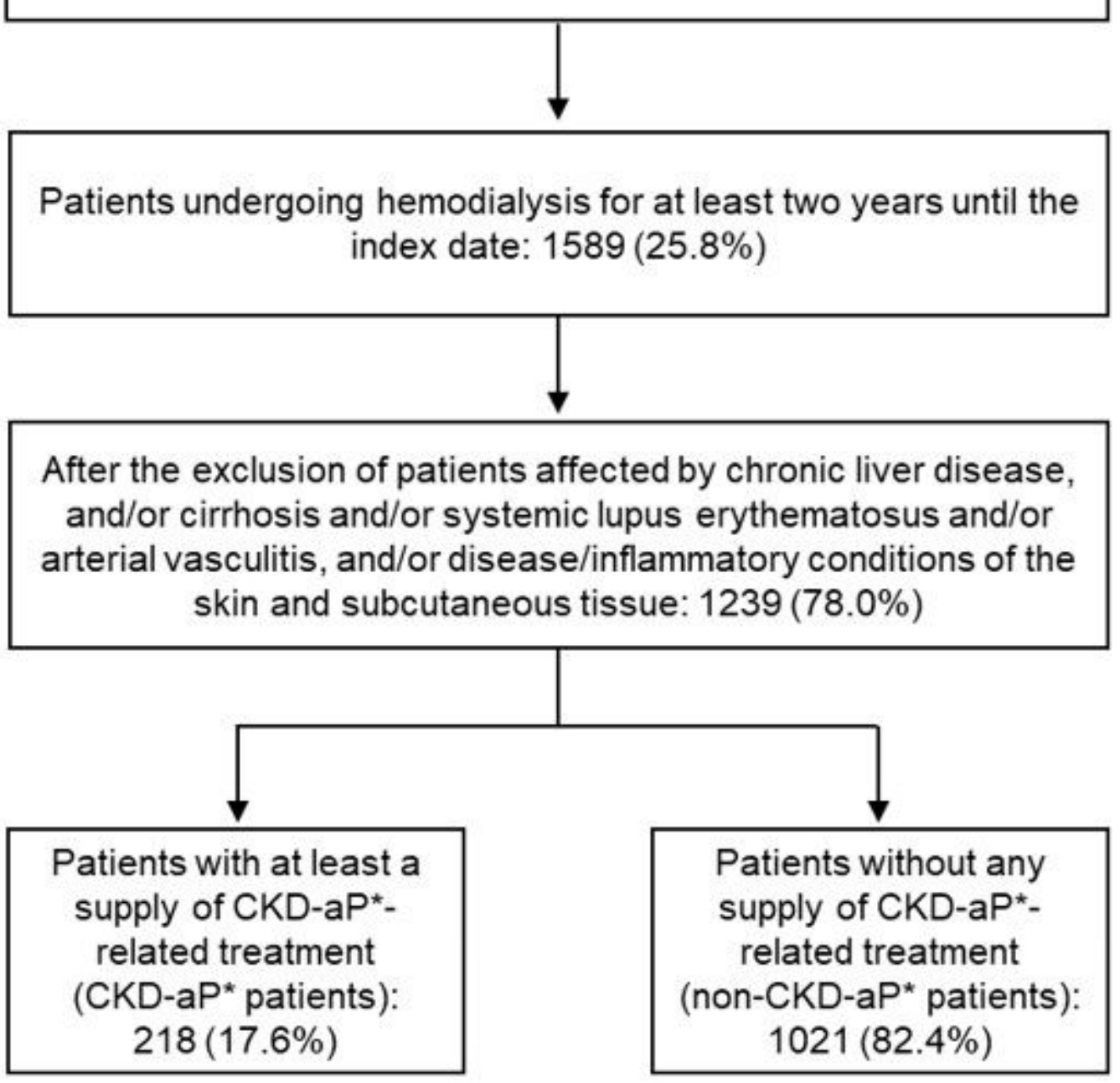

${ }^{*} \mathrm{CKD}$-aP: chronic kidney disease-associated pruritus

\section{Figure 1}

Selection of subjects undergoing hemodialysis and potentially affected or not by chronic kidney diseaseassociated pruritus.

\section{Supplementary Files}

This is a list of supplementary files associated with this preprint. Click to download.

- SupplementarymaterialBMCNephrology2021.pdf 